\title{
Convexity-like inequalities for averages in a convex set
}

\section{Mihai Dragomirescu and Constantin IVAN}

For a function $f: X \rightarrow R \cup\{+\infty\}$, convex and finite over an algebraically open convex subset $C$ of a linear space $X$, we provide a simple necessary and sufficient condition for the "convexity inequality"

$$
f\left(\sum \lambda_{i} x_{i}\right) \leqslant \sum \lambda_{i} f\left(x_{i}\right)
$$

to be satisfied for every integer $n \geqslant 2$ and all $x_{1}, \ldots, x_{n} \in X$ and $\lambda_{1}, \ldots, \lambda_{n} \in R_{+}$ such that $\sum \lambda_{i}=1$ and

$$
\sum \lambda_{i} x_{i} \in C
$$

(i.e. if just the average $\sum \lambda_{2} x_{i}$ lies in the set $C$ of convexity, not the points $x_{1}, \ldots$, $x_{n}$ themselves).

Our condition is simply that $f$ majorizes a certain convex function, depending only on the restriction $f_{C}$ of $f$ to $C$, namely the smallest convex extension of $f_{C}$, which is given explicitly.

We apply this result to enlarge the set of $n$-tuples $\left(x_{1}, \ldots, x_{n}\right) \in X^{n}$ satisfying $\left(\mathrm{C}_{n}\right)$, beyond $C^{n}$, the domain ensured by the convexity of $f$ on $C$. A number of examples show that such an extension of the domain of validity of $\left(\mathbf{C}_{n}\right)$ can be easily obtained when $X=R$.

\section{Sur les solutions globales de l'équation des cocycles}

\section{MOHAMED HMISSI}

Let $\Phi: \mathbb{R}^{+} \times X \mapsto X$ be a semidynamical system and $f: \mathbb{R}^{+} \times X \mapsto \mathbb{R}^{+}$be a cocycle of $(X, \Phi)$, i.e. $f$ verifies the following functional equation:

$$
\forall x \in X ; s, t \geqslant 0: \quad f(s+t, x)=f(t, x) f(s, \Phi(t, x)) .
$$

Under convenient conditions, we give an explicit form of the globally measurable or continuous solutions of (E) and we study the particular case when $X=\mathbb{R}$. 